\title{
Accurate Wideband Evaluation of the Shielding Effectiveness of Complex Enclosures Using an Asynchronous Parallel NSPWMLFMA
}

\author{
Joris Peeters $^{1}$, Ignace Bogaert ${ }^{2}$, Jan Fostier ${ }^{3}$, Femke Olyslager IEEE Fellow ${ }^{4}$ \\ ${ }^{1}$ Ghent University, Department of Information Technology, Ghent, Belgium, joris.peeters@intec.ugent.be \\ ${ }^{1}$ Ghent University, Department of Information Technology, Ghent, Belgium, ignace.bogaert@intec.ugent.be \\ ${ }^{3}$ Ghent University, Department of Information Technology, Ghent, Belgium, jan.fostier@intec.ugent.be \\ ${ }^{4}$ Ghent University, Department of Information Technology, Ghent, Belgium, femke.olyslager@intec.ugent.be
}

\begin{abstract}
We present the application of the Non-directive Stable Plane Wave Multilevel Fast Multipole Algorithm (NSPWMLFMA) to the simulation of the shielding effectiveness of enclosures with complex fillings. The method is parallelized with an asynchronous algorithm in order to allow highly efficient simulations in an inexpensive GRID computing environment. The whole method is fully error controlled. Further increased efficiency is obtained by using Block-Jacobi preconditioners, splay trees (STs) to extract symmetries in the geometry and careful evaluation of selfpatch and neighbor-patch integrals. Numerical examples of enclosures with and without equipment illustrate the method. We will also focus on the use of lossy materials to increase the shielding efficiency of metal enclosures around resonance frequencies.
\end{abstract}

\section{INTRODUCTION}

In [1] a detailed study was made on the shielding performance of metallic enclosures. The simulation of the shielding effectiveness of an enclosure remains a difficult task because of two main reasons. First, the accuracy of the simulations needs to be very high. This requires high precision calculations and very fine discretizations both leading to high computational costs. Second, every geometric detail in the enclosure is of importance. Taking into account this detail again puts high demands on the computational complexity. In [1] a Method of Moments (MoM) based code [2] was used in order to achieve the required accuracy. The high computational cost limited the simulations to low frequencies and simple geometries.

We have already shown that the Multilevel Fast Multipole Algorithm (MLFMA) [3] is especially suited for evaluation of the shielding performance of enclosures. This method combines a high accuracy with limited computational cost. If $N$ represents the number of unknowns to discretize the unknown (equivalent) electric and magnetic current densities on surfaces then the computational cost of the MLFMA is $O(N \log (N))$. For threedimensional problems, involving considerable geometrical detail and/or of several wavelengths in size, $N$ will grow rapidly requiring considerable computer resources even when using MLFMA.

Two-dimensional simulations of shielding enclosures using a boundary integral equation accelerated with MLFMA were presented in [4] and [5]. In these contributions we demonstrated the importance of the accuracy in self-patch and neighbor-patch integrations, the importance of preconditioning and the use of STs to efficiently extract symmetry. In [6] we presented an asynchronous parallelized MLFMA that allows for efficient parallelization of the MLFMA method on inexpensive GRID computer environments connected by a slow switch. The asynchronous algorithm is also highly performant compared to existing synchronous parallelizations of the MLFMA when considering multiple object scattering as is encountered in the evaluation of shielding enclosures comprising realistic hardware. An implementation of this twodimensional parallel MLFMA is available as open source software from [7]. Applications of this method reach far beyond EMC problems, see e.g. [8].

In [6] we already mentioned the possibility to extend this algorithm to three dimensions by considering elementary three-dimensional scattering examples. This paper wants to further explore this and show its abilities to evaluate realistic shielding problems. We apply the MLFMA on different surface integral equations to calculate the scattering at multiple homogeneous dielectric or perfectly conducting (PEC) objects. Since the classical plane wave based MLFMA breaks down at low frequencies it is not very suitable to simulate objects with sub-wavelength detail. However, recently a very efficient new plane wave based MLFMA, the NSPWMLFMA, has been developed [9] that remains stable at low frequencies. In [9] NSPWMLFMA was developed for scalar wave propagation problems, here we for the first time apply it for vectorial wave propagation.

The asynchronous parallelization scheme developed in [6] for two-dimensional problems is shown to be fully applicable for the three-dimensional NSPWMLFMA as will be illustrated here for the first time. As in the two-dimensional case we will devote special attention to preconditioning, accurate evaluation of self-patch and neighbor-patch integrations and the use of STs. In particular the performance in three-dimensions of STs [10] deserves special attention.

In [1] it was argued that shielding at resonance frequencies of enclosures benefits from the use of absorbing materials. We will show that the asynchronous parallel NSPWMLFMA is capable to include lossy objects without losing accuracy. As we did for the two-dimensional implementation we also made this three-dimensional implementation available as open-source software from [7].

The scope of this paper does not allow us to give a detailed description of all the elements of the asyn- 
chronous parallel NSPWMLFMA. We will suffice with a brief discussion of each of these elements with the relevant references, where possible, and rather focus on the simulation results. We will compare results with results obtained in [1], show results of the shielding efficiency of a typical personal computer tower and illustrate the effect of absorbing materials. More results will be shown during the presentation at the conference.

\section{THEORY}

\section{A. Surface integral equations}

The geometry that we consider consists of a number of homogeneous isotropic dielectric objects, each characterized by its complex permittivity and permeability, and of PEC objects both embedded in an isotropic background, usually free space. Objects can be embedded into other objects and objects can touch each other creating lines where three or more materials come together.

The scattering at such geometries is evaluated by surface integral equations with as unknowns the (equivalent) electric and magnetic surface current densities on the boundaries of each object. Several integral equations are possible, for an overview we refer to [12]. We use a combination of different types of integral equations depending on the type of objects and the frequencies involved. For open PEC objects, such as enclosures with apertures, we use the Electric Field Integral Equation (EFIE), for closed objects we use EFIE below the first resonance frequency of the object and the Combined Field Integral Equation (CFIE) above that frequency. For very low frequencies we resort to the Magnetic Field Integral Equation (MFIE) in stead of EFIE because it is better conditioned at low frequencies. For dielectrics we use the Müller integral equation at low frequencies and the Poggio-Miller-ChangHarrington-Wu-Tsai (PMCHWT) formulation at high frequencies.

\section{B. Method of Moments}

We use a Galerkin MoM where the surface current densities are discretized on a triangular mesh with RaoWilton-Glisson vectorial basis functions. To achieve high accuracy the singular part of the Green functions is extracted for both the self-patch and neighbor-patch integrations. These integrations over the singular part are all being done in closed form. The remaining regular part of the Green function and the integrations for interactions that are nor self-patch nor neighbor-patch are done numerically using Gaussian quadrature rules defined on a triangle [11].

Special care is taken when objects are touching. In the past two touching objects were often simulated by including a very small gap between them. While this simplifies the implementation and does not lead to significant error, it does involve twice as many unknowns along the touching surface as strictly necessary. We opted for a more cumbersome implementation, as described in [13], that avoids including these gaps and extra unknowns and that at the same time remains a full Galerkin scheme, which avoids half basis functions at lines where three or more materials come together.

\section{Splay Trees}

Often large structures contain symmetries where two pairs of interacting triangles are geometrically equal which means that the corresponding two elements in the MoM matrix are equal. In [5] we have shown for twodimensional problems that a ST [10] allows to extract these geometrical symmetries for the near interactions in $O(N \log N)$ time complexity, making it compatible with the MLFMA. Also for three-dimensional problems the ST method can be used efficiently, again leading to an $O(N \log N)$ complexity. Because the near interactions are relatively more important in three than in two dimensions, the savings by using STs are even more profound. Even for structures with limited symmetry some savings are seen which shows that the overhead imposed by unsuccessful search operations is limited. The extra memory needed for the ST is only needed during the set-up phase of the MLFMA.

\section{NSPWMLFMA}

The classical plane wave based MLFMA breaks down at low frequencies due to loss of numerical accuracy. This means that the boxes on the lowest level in the MLFMA for structures containg significant sub-wavelength geometrical detail will contain a considerable number of unknowns. Several attempts have been proposed to remedy this problem without having to resort to non-diagonal translation operators. The crux is to incorporate more near-field information in the plane wave spectrum. This can be done by incorporating evanescent plane waves leading to the Stable Plane Wave MLFMA [14] which needs 6 radiation patterns along 6 different directions. Recently a new method, the NSPWMLFMA, was devised [9] that avoids these 6 different directions for scalar wave propagation problems. In this contribution we for the first time report results obtained with the NSPWMLFMA for vectorial wave propagation. The NSPWMLFMA is easily incorporated in and is fully compatible with the classical MLFMA.

\section{E. Asynchronous parallelization}

In [6] it was announced that the asynchronous parallelization developed for two-dimensional MLFMA was mutatis-mutandis applicable for the three-dimensional case. In this contribution we, for the first time, present results obtained in three dimensions. The asynchronous parallelization uses a space filling curve to assign different boxes in the MLFMA to different processors. The workload is then divided in small packets which are arranged in a priority queue. The priority queue is built using an advanced heuristic and avoids communication bursts between processors as would be the case in synchronous parallelization. This makes this method highly suitable for low cost GRID computing environments connected by standard gigabit Ethernet. It is also very favourable when scattering at multiple objects is considered as is the case for shielding effectiveness evaluations.

\section{SHIELDING PROBLEMS}

As a verification we first consider the cubical brass box of dimensions $50 \mathrm{~cm} \times 50 \mathrm{~cm} \times 50 \mathrm{~cm}$ that was also 


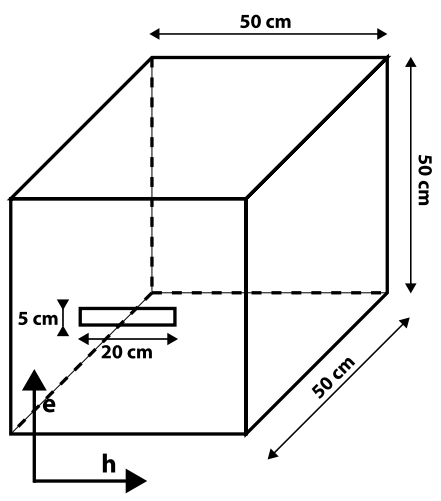

Fig. 1. Cubic box with slit illuminated by a plane wave.

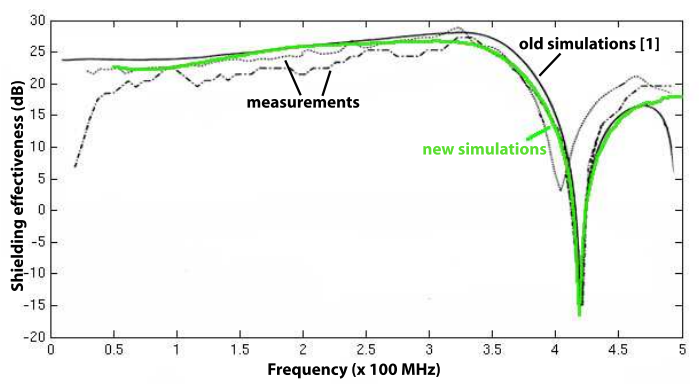

Fig. 2. Shielding effectiveness of the box in Fig. 1.

extensively investigated in [1]. The front panel of the box is interchangable and here we will consider a front panel with a slit of $5 \mathrm{~cm} \times 20 \mathrm{~cm}$ in the centre of the box. As a reference solution we consider a box without a front panel. The box is perpendicularly illuminated by a plane wave with the electric field polarized orthogonal to the slit. The electric field is measured in the centre of the box. The set-up is shown in Fig. 1.

Figure 2 shows the shielding efficiency of the box when the box without front panel is used as reference. As a comparison also results from [1] are shown which consist both of measurements and simulations. As can be seen the new simulations are somewhat closer to the measurements than the simulations in [1]. This is due to the fact that the number of unknowns $N=11744$ is about ten times higher than was possible in [1].

In [1] it was mentioned that the application of absorbing materials in a shielding enclosure could help improving the shielding efficiency around resonance frequencies. To illustrate this effect we place an absorbing plate of $46 \mathrm{~cm}$ $\times 46 \mathrm{~cm} \times 2 \mathrm{~cm}$ at a distance of $2 \mathrm{~cm}$ from the bottom in the cubic enclosure. Horizontally the plate is centred in the box. The complex relative permittivity of the plate is $2-2 j$. As a comparison we also consider a lossless plate with the same dimensions but with a relative permittivity of 2. The entire structure is meshed using $N=38951$ unknowns. Figure 3 shows the shielding efficiency as a function of frequency with lossy plate, with lossless plate and without plate. The reference always is the enclosure with open front and without plate. We note as expected an increase in shielding efficiency around resonances. Also

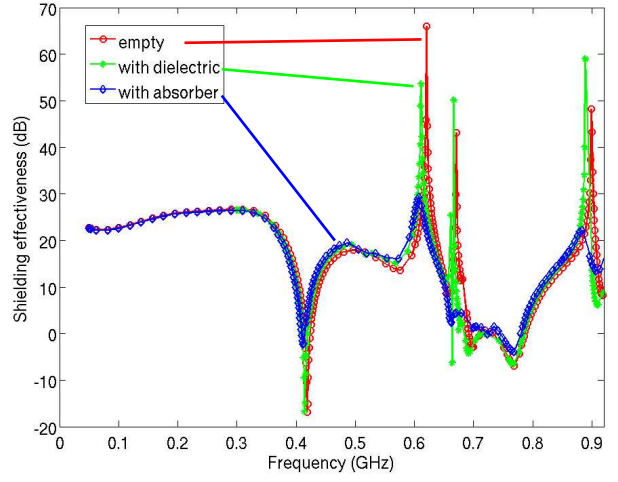

Fig. 3. Shielding effectiveness of the box in Fig. 1 with a lossy plate (diamonds), with a lossless plate (stars) and without a plate (circles).
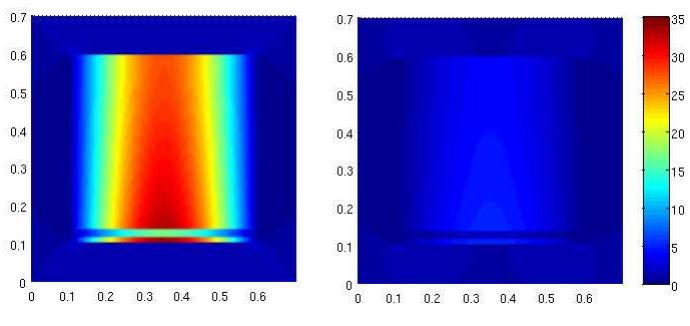

Fig. 4. Field distribution at first resonance in the box of Fig. 1 with lossless (left) and lossy (right) plate.

note that the losses of the plate indeed are responsible for an increased shielding efficiency. In Fig. 5 the amplitude of the vertical component of the electric field is shown in a vertical cross-sectional plane of the enclosure at the first resonance. The figure on the left corresponds with the lossless plate and the figure on the right with the lossy plate. Note the substantial decrease in field amplitudes inside the enclosure due to the losses.

As a final example we consider a personal computer filled with a number of objects and a number of holes in the casing. Figures 5 and 6 give an idea of the mesh which resulted in $N=44852$ unknowns. With $N=44852$ the problem was simulated on 16 processors ( 8 dual core 2GHz AMD 64 bit processors connected by a 1 GBit Ethernet switch) using 3.2 GByte of memory when using STs and 1.6 GByte without using STs. At $250 \mathrm{MHz}$ the setup time reduced from 960 s to $526 \mathrm{~s}$ due to the use of STs. The NSPWMLFMA required $0.5 \mathrm{~s}$ per iteration for a total of 251 iterations. Fig. 7 shows the shielding efficiency as a function of frequency when considering an incident plane wave with the electric field polarized along the height of the case. Three different discretizations are considered resulting in $N=15495, N=33394$ and $N=66980$. The small difference in shielding efficiency at low frequencies is due to a reduction of numerical leakage through the walls when using finer meshes. The amplitude of the vertical electric field component in the central vertical cross-section at a resonance at $1 \mathrm{GHz}$ is shown in Fig. 8. 

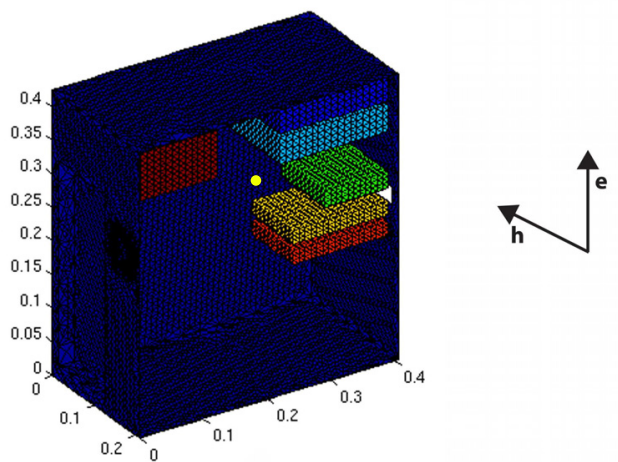

Fig. 5. Internal view of a personal computer. Dimensions are in $\mathrm{m}$ and the yellow dot indicates the measure point.
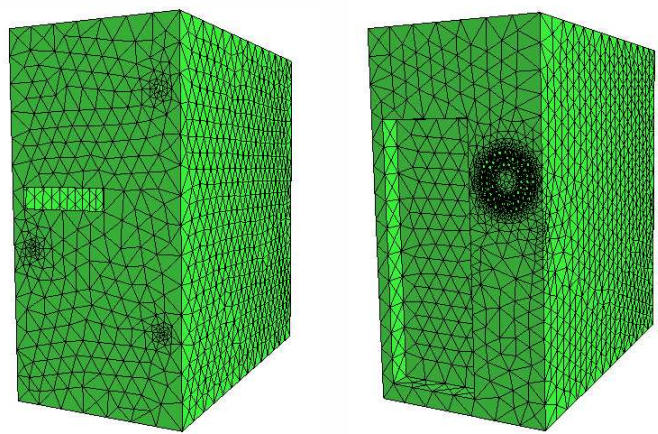

Fig. 6. Front (left) and rear (right) view of the external mesh of a personal computer.

\section{CONCLUSIONS}

We demonstrated the use of a non-directional plane wave based MLFMA stable at low frequencies for the simulation of complex shielding problems. An advanced asynchronous parallel implementation of the MLFMA allows for efficient simulations on inexpensive GRID computing environments. Further research will focus on better preconditioners to reduce the number of iterations and towards further acceleration of the algorithms in order to be able to simulate even more complex structures with reasonable computational effort.

\section{REFERENCES}

[1] F. Olyslager, E. Laermans, D. De Zutter, S. Criel, R. De Smedt N. Lietaert, and A. De Clercq, "Numerical and experimental study of the shielding effectiveness of a metallic enclosure," IEEE Trans. Electromagn. Compat., vol. EMC-41, no. 3, pp. 202-213, Aug. 1999.

[2] T. Mader and H.D. Brüns, "EFIE analysis of arbitrary metallic structures in the area of electromagnetic compatibility," in 9th Int. Symp. Electromagnet. Compat., Zürich, Switzerland, Mar. 1991

[3] W.C. Chew, J.M. Jin, E. Michielssen, and J. Song, Fast and Efficient Algorithms in Computational Electromagnetics, Boston: Artech House, 2001.

[4] F. Olyslager and J. Fostier, "Fast and accurate evaluation of the shielding effectiveness of complex enclosures," in Proc. 17th Int. Zurich Symp. on Electromagnet. Compat., Singapore, pp. 30-33, Feb. 2006.

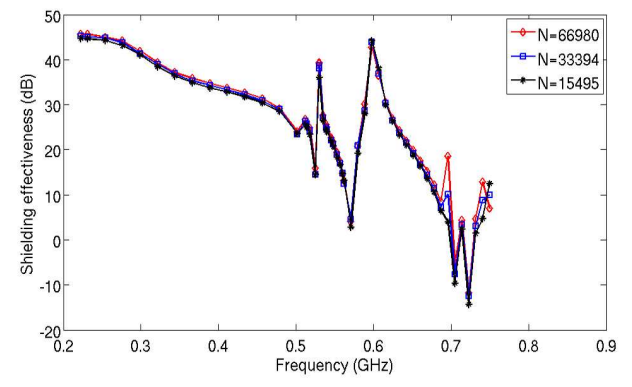

Fig. 7. Shielding effectiveness of the enclosure of Figs. 5 and 6.

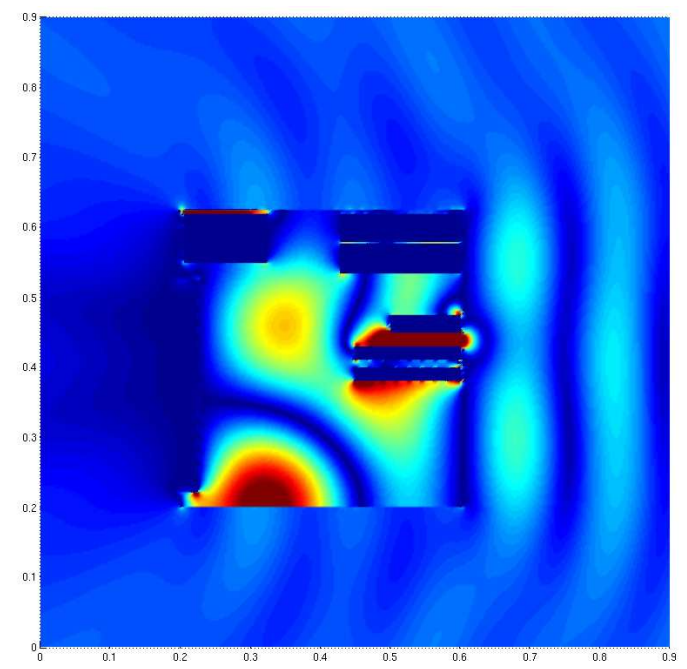

Fig. 8. Amplitude of the vertical electric field component in the cross section of the enclosure of Figs. 5 and 6 at $250 \mathrm{MHz}$.

[5] F. Olyslager, J. Fostier, J. Peeters, and D. De Zutter, "Fast and accurate evaluation of enclosures with the method of moments by using splay trees," in Proc. 18th Int. Zurich Symp. on Electromagnet. Compat., Munich, pp. 87-90, Sept. 2006.

[6] J. Fostier and F. Olyslager, "An asynchronous parallel MLFMA for scattering at multiple dielectric objects," Provisionally accepted for IEEE Trans. on Antennas and Propagat.

[7] J. Fostier, J. Peeters, and F. Olyslager, "Open FMM", [Online]. Available: http://openfmm.intec.ugent.be.

[8] J. Fostier and F. Olyslager, "A GRID computer implementation of the multilevel fast multipole algorithm for full-wave analysis of optical devices," IEICE Trans. on Com., Vol. E90-B, no. 9, pp. 2430-2438, Sept. 2007.

[9] I. Bogaert, J. Peeters, and F. Olyslager, "A nondirective plane wave MLFMA stable at low frequencies," Submitted for IEEE Trans. on Antennas and Propagat. .

[10] D.D. Sleator and R.E. Tarjan, "Self-adjusting binary search trees," J. of the ACM, Vol. 32, pp. 652-686, July 1985.

[11] M.A. Taylor, B.A. Wingate, and L.P. Bos, "Several new quadrature formulas for polynomial integration in the triangle," [Online]. Available: http://arxiv.org/PS_cache/math/pdf/0501/0501496v2.pdf, Feb. 2007.

[12] P. Yla-Oijala, M. Taskinen, and S. Jarvenpaa, "Surface integral equation formulations for solving electromagnetic scattering problems with iterative methods," Radio Science, vol. 40, RS6002.

[13] P. Yla-Oijala, M. Taskinen, and J. Sarvas, "Surface integral equation method for general composite metallic and dielectric structures with junctions," PIER 52, pp. 81-108, 2005.

[14] E. Darve and P. Havé, "A fast multipole method for Maxwell equations stable at all frequencies," Phil. Trans. R. Soc. Lond. A, Vol. 362, pp. 603-628, 2004. 